\title{
Adaptive Output Feedback Tracking Controller Design of Stochastic Nonlinear Systems with Parameter Uncertainty for Polynomial Function Growth Conditions
}

\author{
Long-Chuan Guo \\ School of Mechanical Engineering, Hangzhou Dianzi University, Hangzhou 310018, China \\ Correspondence should be addressed to Long-Chuan Guo; glc1988@126.com
}

Received 22 January 2018; Accepted 2 May 2018; Published 19 June 2018

Academic Editor: Francesco Aggogeri

Copyright (c) 2018 Long-Chuan Guo. This is an open access article distributed under the Creative Commons Attribution License, which permits unrestricted use, distribution, and reproduction in any medium, provided the original work is properly cited.

\begin{abstract}
This paper mainly focuses on output feedback practical tracking controller design for stochastic nonlinear systems with polynomial function growth conditions. Mostly, there are some studies on output feedback tracking control problem for general nonlinear systems with parametric certainty in existing achievements. Moreover, we extend it to stochastic nonlinear systems with parametric uncertainty and system nonlinear terms are assumed to satisfy polynomial function growth conditions which are more relaxed than linear growth conditions or power growth conditions. Due to the presence of unknown parametric uncertainty, an output feedback practical tracking controller with dynamically updated gains is constructed explicitly so that all the states of the closedloop systems are globally bounded and the tracking error belongs to arbitrarily small interval after some positive finite time. An example illustrates the efficiency of the theoretical results.
\end{abstract}

\section{Introduction}

The problem of global output tracking control for nonlinear systems has been extensively studied by academic researchers and successfully applied in some practical nonlinear systems (see [1-10]). According the existing papers, two types output tracking have been concerned which are asymptotic output tracking and practical tracking (see [11-16]). The asymptotic output tracking focuses on the design of controller that forces the controlled output of system to reach and follow a timeparameterised reference track signal. However, for many practical systems and control design, we can hardly achieve good models, too expensive to derive, and the parameters are not precisely known preventing the error signals from tending to zero, so the practical tracking has been proposed by many researchers.

Consider the output feedback practical tracking control problem for a class of nonlinear system which appears in past research papers:

$$
\begin{aligned}
& d x_{i}=x_{i+1} d t+f_{i}(x) d t \quad i=1, \cdots, n-1 \\
& d x_{n}=u d t+f_{n}(x) d t
\end{aligned}
$$

$$
y=x_{1}-y_{r}
$$

where $x=\left(x_{1}, \ldots, x_{n}\right)^{T}, u \in R$, and $y \in R$ are the system states, input, and output. Correspondingly, $y_{r}$ is given reference trajectory, and the nonlinear term $f_{i}(x)$ satisfies the Lipschitz condition, whose initial value is $f_{i}(0)=0$. Some related papers have studied the practical output feedback control problem for nonlinear system (1) (see [17-22]). In [17, 18], the system nonlinear term satisfies lower triangular structure and is limited to the following order of polynomial function growth conditions:

$$
c_{0}\left(1+\left|x_{1}\right|^{p}\right)\left(\left|x_{1}\right|+\cdots+\left|x_{i}\right|\right)+c_{0}
$$

where $p \geq 1$ and $c_{0} \geq 0$ are known constants. Similarly, the system nonlinear term satisfies the following growth conditions which have contained known constants $c$ and $\tau$ in [22]:

$$
\begin{aligned}
& c\left(\left|x_{1}\right|^{i \tau+1}+\left|x_{2}\right|^{(i \tau+1) /(\tau+1)}+\cdots+\left|x_{i}\right|^{(i \tau+1) /((i-1) \tau+1)}\right) \\
& \quad+c
\end{aligned}
$$


After introducing the unknown parameters into the growth condition of system (1), [19] has studied output feedback tracking control research on this kind of uncertain nonlinear system by introducing coordinate transformation, using Nussbaum gain method and adding power integral method so as to obtain a continuous adaptive controller and ensure that system tracking error converges to a small neighborhood of zero after a limited time, while the closed-loop nonlinear system meets the boundedness of the states. In [20], an adaptive output feedback tracking controller is designed by means of general control and dead zone method. It is pointed out that the nonlinear term growth condition of the system in [20] depends on the unmeasurable states and the growth rate is unknown constant; meanwhile, the reference trajectory signal and its first derivative have unknown upper bound.

However, all the mentioned references are only limited to the nonlinear systems. Naturally, one may ask an interesting and challenging question: If we consider the tracking control problem for stochastic nonlinear system, how to design a controller? As is well known, stochastic factor is a main resource that contributes significantly to the system complexity. In some practical fields, such as aerospace, biological, and economic systems and health community, the output tracking problem for these stochastic nonlinear systems has a wide range of practical applications. The treatment of stochastic items is the key to solving our problems in the stochastic control system. To our knowledge, the existing research results on the tracking problem for stochastic nonlinear systems are [23-27]. The work [23] has utilized a backsteppingbased control for a class of stochastic nonlinear systems where the controller has ensured the overall system stability. The works [24-27] further consider the states feedback tracking controller design problem for high-order stochastic nonlinear system, satisfying a more relax condition. As is known to all, output feedback controller is more practical than state feedback controller in the actual system because states information of actual system is difficult to master. For the stochastic nonlinear system where nonlinear vector fields depend on the unmeasurable states besides the measurable output, how to design an output feedback tracking controller? This paper is focused on the problem of extending the results of $[25,27]$ which has not yet been studied till now.

Meanwhile, some research has studied output feedback practical tracking control problem for a class of stochastic nonlinear system which satisfies the growth condition of polynomial function, the nonlinear term of the system depends on the state of unmeasurable, and the growth condition of the nonlinear term parameters is known (see $[17,18]$ ). This leads to some limitations; since parameter uncertainties and structural uncertainties are common in practical systems such as spaceflight control systems, navigation systems, and bioengineering systems, this is far from sufficient for research on output feedback tracking control problem for stochastic nonlinear system under known parameters conditions.

This paper extends it to stochastic nonlinear systems and proposes to set the corresponding parameters of stochastic nonlinear system to be unknown and introduce the parameter uncertainty factors, so as to generalize the output feedback practical tracking control research results in the previous papers. The main contribution of this paper is as follows.

Use Ito differential mathematics theory to deal with complex stochastic terms such that a high-order gain observer and trajectory tracking error system are constructed. Then the error system is analyzed by Lyapunov function and the boundedness of system parameters is proved. The output feedback practical tracking controller with dynamically updated gains is constructed explicitly so that all the states of the closed-loop system are bounded and the error converges to any small neighborhood of the origin when the time goes to infinity.

The paper is organized as follows. Section 2 provides some problem description. In Section 3, an output feedback practical tracking controller is designed and analyzed. A simulation example is provided in Section 4. Section 5 concludes this paper.

\section{Problem Description}

In this paper, we consider the output feedback practical tracking problem for following stochastic nonlinear system:

$$
\begin{aligned}
d x_{i} & =x_{i+1} d t+f_{i}(x) d \omega \quad(i=1, \cdots, n-1) \\
d x_{n} & =u d t+f_{n}(x) d \omega \\
y & =x_{1}-y_{r}(t)
\end{aligned}
$$

where $x=\left(x_{1}, \cdots, x_{n}\right)^{T} \in R^{n}$ and $u \in R$ are the system states and input, $y$ is the system output that can be measured, respectively, $y_{r}$ is given reference tracked unmeasured trajectory, similarly, $x_{2}(t), \ldots, x_{n}(t)$ are unmeasurable, and $\omega$ is an $m$-dimensional standard Wiener process defined on the complete probability space $(\Omega, \Gamma, \mathrm{P})$ with $\Omega$ being a sample space, $\Gamma$ being a filtration, and $\mathrm{P}$ being a probability measure. The nonlinear functions $f_{i}: R^{+} \times R^{n} \rightarrow R^{m}, i=1, \cdots, n$, are continuous and assumed to satisfy locally Lipschitz condition with $f_{i}(0)=0$.

According to the above description of system, it leads to the objective of this paper: for any constant $\delta>0$, all the states of stochastic nonlinear system (4) are well defined and bounded. In addition, there exists a finite time $T>0$ such that, for any $t>T$, it can get

$$
\left|x_{1}(t)-y_{r}(t)\right| \leq \delta, \quad \forall t \geq T
$$

To achieve the above control performance, the following assumptions are made for the nonlinear term in system (4).

Assumption 1. There exist unknown constants $\eta_{1}>0, \eta_{2}>0$ and known integer $p \geq 1$ such that the following inequality holds:

$$
\left|f_{i}(x)\right| \leq\left(1+\left|x_{1}\right|^{p}\right)\left(\eta_{1}\left(\left|x_{1}\right|+\cdots+\left|x_{i}\right|\right)+\eta_{2}\right)
$$

Next traced trajectory $y_{r}$ satisfies the following assumption.

Assumption 2. The reference output trajectory $y_{r}$ of stochastic nonlinear system (4) is continuously differentiable and 
there exists unknown constant $\eta>0$ such that the following inequality holds:

$$
\left|y_{r}\right|+\left|\dot{y}_{r}\right| \leq \eta
$$

Assumption 1 can be further extended to the following more general assumption conditions.

Assumption 3. There exist unknown constants $c>0, d>0$ $(d / c \geq 1)$ and known constants $p \geq 1,0 \leq \mu \leq 1$, such that the following inequality holds:

$$
\begin{aligned}
& s^{0}\left|f_{1}(x)\right|+\cdots+s^{n-1}\left|f_{n}(x)\right| \\
& \quad \leq\left(1+\left|x_{1}\right|^{p}\right)\left(c\left(s^{0}\left|x_{1}\right|+\cdots+s^{n-1}\left|x_{n}\right|\right)+d\right)
\end{aligned}
$$

where $s(0 \leq s \leq \mu)$ is any constant.

This paper will focus on stochastic nonlinear system under more general Assumption 3 and then construct an output feedback practical tracking controller such that output of system (4) can be gradually converged to zero.

\section{System Analysis and Output Feedback Controller Design}

The emphasis of this section is to design the global output feedback tracking controller in order to achieve asymptotic stability of system output (4) under Assumptions 2 and 3 .

Remark 4. From Assumption 3, we can clearly see that the unknown parameter $c>0$ appears in the product term in the front of unmeasurable states $\left(x_{2}, \cdots, x_{n}\right)$, which leads to the fact that the conventional observer design method difficultly works because the constructed observer contains initial states of the system which contains unknown parameters such that the traditional method is no longer applicable. Inspired by $[21,22]$, this paper develops a new type of output tracking controller which consists of two dynamic factors, and it will be described in detail below.

3.1. Time Varying High-Order Gain Observer Design. First, make a general assumption as follows:

$$
\frac{d}{c} \geq 1
$$

and for the sake of convenience of formula derivation, the following states transformation is introduced:

$$
\begin{gathered}
z_{1}=x_{1}-y_{r} \\
z_{2}=x_{2} \\
\vdots \\
z_{n}=x_{n}
\end{gathered}
$$

and then stochastic nonlinear system (4) is converted to

$$
d z_{i}=z_{i+1} d t+g_{i}(z) d \omega \quad(i=1, \cdots, n-1)
$$

$$
\begin{aligned}
d z_{n} & =u d t+g_{n}(z) d \omega \\
y & =z_{1}
\end{aligned}
$$

where $g_{i}$ is the nonlinear term after conversion and is constructed as follows:

$$
\begin{aligned}
& g_{1}(z)=f_{1}\left(z_{1}+y_{r}, z_{2}, \cdots, z_{n}\right)-\frac{d y_{r}}{d \omega} \\
& g_{i}(z)=f_{i}\left(z_{1}+y_{r}, z_{2}, \cdots, z_{n}\right) \quad(i=2, \cdots, n)
\end{aligned}
$$

Next, the output feedback tracking controller design process is initiated. With unmeasurable states $x_{1}, \cdots, x_{n}$ characteristics of system (4), states observer of system (4) is established

$$
\begin{aligned}
& \dot{\vec{z}}_{i}=\widehat{z}_{i+1}+h_{i}(A B)^{i}\left(z_{1}-\widehat{z}_{1}\right) \quad(i=1, \cdots, n-1) \\
& \dot{\vec{z}}_{n}=u+h_{n}(A B)^{n}\left(z_{1}-\widehat{z}_{1}\right)
\end{aligned}
$$

where $\widehat{z}_{i}$ is the estimate value of $z_{i}$ and the high-order gain term consists of two variables $A(t)$ and $B(t)$ as follows:

$$
\begin{array}{ll}
\dot{A}(t)=\frac{A}{(A B)^{2 b}}\left(\widehat{z}_{1}^{2}+e_{1}^{2}-\frac{1}{2} \delta^{2}\right), & A(0)=A_{0} \geq \frac{1}{\mu} \\
\dot{B}(t)=-\mu_{1} B^{2}+\mu_{2} B\left(1+\left|x_{1}\right|^{p}\right)^{2}, & B(0)=1
\end{array}
$$

where $e_{1}=z_{1}-\widehat{z}_{1}$ is error state, $h_{i}>0(i=1, \cdots, n)$ is the coefficient of the Hurwitz polynomial with $s^{n}+h_{1} s^{n-1}+\cdots+$ $h_{n-1} s+h_{n}$, and the unknown parameters $b, \mu_{1}, \mu_{2}$ satisfy $0<$ $b<1 / 4 p, 0<\mu_{1}<\mu_{2}$.

3.2. Lyapunov Analysis of Closed-Loop Systems. According to (14) on the definition of $e_{1}$, it can be generalized to all states; that is, definition of $e_{i}=z_{i}-\widehat{z}_{i}(i=1, \cdots, n)$ and the available system error equation can be obtained:

$$
\begin{aligned}
& d e_{i}=e_{i+1} d t-h_{i}(A B)^{i} e_{1} d t+g_{i}(z) d \omega \\
& d e_{n}=-h_{n}(A B)^{n} e_{1} d t+g_{n}(z) d \omega
\end{aligned}
$$

In order to facilitate the output feedback practical tracking controller design, the transformation of estimated states $\widehat{z}_{i}$ and error states $e_{i}$ is introduced:

$$
\begin{aligned}
& \alpha_{i}=\frac{e_{i}}{(A B)^{b+i-1}} \quad(i=1, \cdots, n) \\
& \beta_{i}=\frac{\widehat{z}_{i}}{(A B)^{b+i-1}} \quad(i=1, \cdots, n)
\end{aligned}
$$

where $\alpha=\left(\alpha_{1}, \cdots, \alpha_{n}\right)^{T}, \beta=\left(\beta_{1}, \cdots, \beta_{n}\right)^{T}, h=\left(h_{1}, \cdots\right.$, $\left.h_{n}\right)^{T}$. By (16), stochastic nonlinear systems (11) and (13) can be converted to

$$
\begin{aligned}
d \alpha= & A B H \alpha d t-\left(\frac{\dot{A} B+\dot{B} A}{A B}\right) C_{b} \alpha d t \\
& +G(z, A, B) d \omega
\end{aligned}
$$

$$
\dot{\beta}=A B H_{b} \beta+A B h \alpha_{1}-\left(\frac{\dot{A} B+\dot{B} A}{A B}\right) C_{b} \beta
$$


where

$$
\begin{aligned}
H & =\left(\begin{array}{cccc}
-h_{1} & 1 & \cdots & 0 \\
\vdots & \vdots & \ddots & \vdots \\
-h_{n-1} & 0 & \cdots & 1 \\
-h_{n} & 0 & \cdots & 0
\end{array}\right) \\
H_{b} & =\left(\begin{array}{cccc}
0 & 1 & \cdots & 0 \\
\vdots & \vdots & \ddots & \vdots \\
0 & 0 & \cdots & 1 \\
-h_{1} & -h_{2} & \cdots & -h_{n}
\end{array}\right) \\
C_{b} & =\operatorname{diag}(b, b+1, \cdots, b+n-1) \\
G(z, A, B) & =\left(\frac{g_{1}}{(A B)^{b}}, \frac{g_{2}}{(A B)^{b+1}}, \cdots, \frac{g_{n}}{(A B)^{b+n-1}}\right)^{T}
\end{aligned}
$$

From (14) on the definition of $A(t)$ and $B(t)$, it can be generally obtained that

$$
A(t) \geq A(0) \geq \frac{1}{\alpha} \geq 1, \quad \forall t \geq 0
$$

and defining $E=B^{2}$, the following can be obtained:

$$
\dot{E} \geq 2 \mu_{1} E(1-\sqrt{E}), \quad E(0)=1
$$

According to the comparison lemma in [28], the following can be drawn:

$$
B(t) \geq 1, \quad 0<\frac{1}{A(t) B(t)} \leq \mu, \quad \forall t \geq 0
$$

By (16), it can be obtained that

$$
z_{i}=(A B)^{b+i-1} \alpha_{i}+(A B)^{b+i-1} \beta_{i}
$$

and according to (21)-(22) and Assumptions 2 and 3, the inequality for nonlinear $G(z, A, B)$ can be deduced:

$$
\begin{aligned}
& |G(z, A, B)| \\
& \quad=\left(\left(\frac{g_{1}}{(A B)^{b}}\right)^{2}+\cdots+\left(\frac{g_{n}}{(A B)^{b+n-1}}\right)^{2}\right)^{1 / 2} \\
& \quad \leq\left(1+\left|x_{1}\right|^{p}\right)\left(c \sum_{i=1}^{n} \frac{1}{(A B)^{b+i-1}}\left|z_{i}\right|+c \eta+d\right) \\
& \quad \leq\left(1+\left|x_{1}\right|^{p}\right)(|\alpha|+|\beta|+1)\left(c\left(\eta+\frac{d}{c}\right)\right)
\end{aligned}
$$

where $\bar{c}=c(\eta+d / c)$.

Then a systematic Lyapunov analysis is performed. First, the existence and uniqueness of the system solution are given where, since closed-loop stochastic nonlinear system (17) is continuous and satisfies locally Lipschitz condition on the initial point, the corresponding solution $(\alpha, \beta, A, B)$ of the system can be considered as existence and uniqueness.

Define the following Lyapunov function:

$$
V_{0}(\alpha, \beta)=\alpha^{T} P \alpha+\beta^{T} Q \beta
$$

and positive definite matrices $P, Q$ satisfy the following relation:

$$
\begin{aligned}
H^{T} P+P H & \leq-I_{n} \\
r_{1} I_{n} & \leq C_{b} P+P C_{b} \leq r_{2} I_{n} \\
H_{b}^{T} Q+Q H_{b}^{T} & \leq-2 I_{n} \\
r_{3} I_{n} & \leq C_{b} Q+Q C_{b} \leq r_{4} I_{n}
\end{aligned}
$$

where $r_{i}(i=1,2,3,4)$ is positive constant and $I_{n}$ is $n$ dimensional judgment matrix.

By (23), the following can be obtained:

$$
\begin{aligned}
& \left|2 \alpha^{T} P G\right| \\
& \leq 2\|P\|\left(c\left(\eta+\frac{d}{c}\right)\right)\left(1+\left|x_{1}\right|^{p}\right)(|\alpha|+1)(|\alpha|+|\beta|) \\
& \leq\left(1+\left|x_{1}\right|^{p}\right)^{2}\left(|\alpha|^{2}+|\beta|^{2}\right)+\eta_{1}\left(|\alpha|^{2}+1\right)
\end{aligned}
$$

where $\eta_{1}=(c(\eta+d / c))^{2}\|P\|^{2}$ is unknown constant.

Further, by (25) and (26), the trajectory of (24) along the Ito differentiation for nonlinear stochastic system (17) can be obtained as follows:

$$
\begin{aligned}
L V_{0} & (\alpha, \beta) \\
\leq & -A B|\alpha|^{2}\left(\frac{\dot{A} B+\dot{B} A}{A B}\right)\left(\alpha^{T}\left(C_{b} P+P C_{b}\right) \alpha\right) \\
& +\operatorname{Tr}\left(G^{T} P G\right)-2 A B|\beta|^{2}+2 A B \alpha_{1} \beta^{T} h \\
& -\left(\frac{\dot{A} B+\dot{B} A}{A B}\right)\left(\beta^{T}\left(C_{b} Q+Q C_{b}\right) \beta\right) \\
\leq & -A B|\alpha|^{2}-2 A B|\beta|^{2} \\
& +\|P\|\left(1+\left|x_{1}\right|^{p}\right)^{2}(|\alpha|+|\beta|+1)^{2}\left(c\left(\eta+\frac{d}{c}\right)\right)^{2} \\
& -\left(\frac{\dot{A} B+\dot{B} A}{A B}\right)\left(r_{2}|\alpha|^{2}+r_{4}|\beta|^{2}\right)+2 \alpha^{T} P G \\
& +2 A B \alpha_{1} \beta^{T} Q h \\
\leq & -A B|\alpha|^{2}-2 A B|\beta|^{2} \\
& +\|P\|\left(1+\left|x_{1}\right|^{p}\right)^{2}(|\alpha|+|\beta|+1)^{2}\left(c\left(\eta+\frac{d}{c}\right)\right)^{2} \\
& -\left(\frac{\dot{A} B+\dot{B} A}{A B}\right)\left(r_{2}|\alpha|^{2}+r_{4}|\beta|^{2}\right)
\end{aligned}
$$




$$
\begin{aligned}
& +\left(1+\left|x_{1}\right|^{p}\right)^{2}\left(|\alpha|^{2}+|\beta|^{2}\right)+\eta_{1}\left(|\alpha|^{2}+1\right) \\
& +A B\left(|\alpha|^{2}+|\beta|^{2}\right) \\
\leq & -A B|\alpha|^{2}-2 A B|\beta|^{2}+\eta_{1}\left(|\alpha|^{2}+1\right) \\
& +\mu_{1} B\left(r_{2}|\alpha|^{2}+r_{4}|\beta|^{2}\right) \\
& -\left(1+\left|x_{1}\right|^{p}\right)^{2}\left(|\alpha|^{2}+|\beta|^{2}\right) \\
& +\eta_{1}\left(1+\left|x_{1}\right|^{p}\right)^{2}(|\alpha|+|\beta|+1)^{2} \\
\leq & -B\left(A-\left(2 \eta_{1}+\mu_{1} r_{2}+\mu_{1} r_{4}\right)\right)\left(|\alpha|^{2}+|\beta|^{2}\right)+\eta_{1}
\end{aligned}
$$

and Ito differentiation results after simplifying can be obtained:

$$
\begin{aligned}
L V_{0} \leq & -B\left(A-\eta_{2}\right)\left(|\alpha|^{2}+|\beta|^{2}\right) \\
& +\frac{1}{2}\left(\eta_{2}-\mu_{1}\left(r_{2}+r_{4}\right)\right)
\end{aligned}
$$

where $\eta_{2}=2 \eta_{1}+\mu_{1}\left(r_{2}+r_{4}\right)$. Since matrices $P, Q$ are positive, the following can be easily obtained:

$$
\begin{aligned}
\lambda_{1}\left(\alpha^{T} P \alpha+\beta^{T} Q \beta\right) & \leq|\alpha|^{2}+|\beta|^{2} \\
& \leq \lambda_{2}\left(\alpha^{T} P \alpha+\beta^{T} Q \beta\right)
\end{aligned}
$$

where $0<\lambda_{1} \leq \lambda_{2}$. Moreover, by (29), (28) be deduced into

$$
\begin{gathered}
L V_{0} \leq-\lambda_{1} B\left(A-\eta_{2}\right) V_{0}+\frac{1}{2}\left(\eta_{2}-\mu_{1}\left(r_{2}+r_{4}\right)\right) \\
\text { if }\left(A-\eta_{2} \geq 0\right) \\
L V_{0} \leq-\lambda_{2} B\left(A-\eta_{2}\right) V_{0}+\frac{1}{2}\left(\eta_{2}-\mu_{1}\left(r_{2}+r_{4}\right)\right) \\
\text { if }\left(A-\eta_{2}<0\right)
\end{gathered}
$$

3.3. Boundedness Analysis of System States and Gain. According to (30), it is easy to observe that if $\eta_{2}$ is a known constant, then system (17) can be guaranteed as asymptotically stable by requiring high-gain $A$ to be as large as possible which has been studied by some existing papers. However, in this paper, the parameter $\eta_{2}$ is an unknown constant which requires complex and rigorous analysis to determine the boundedness of the system on the maximum time interval $[0, T)$ and ensure the asymptotical stability of system tracking output.

This section mainly focuses on the boundedness of highgains $A, B$ and system states $\alpha, \beta$. By (14), the following can be obtained:

$$
A(t) \geq \frac{1}{\mu} \quad t \in[0, T)
$$

Accordingly, a hypothetical judgment can be drawn:

$$
\lim _{t \rightarrow T} A(t) \in\left[\frac{1}{\mu},+\infty\right)
$$

To verify the above judgment (32), we make use of the negative proof technology; that is, if conclusion (32) does not hold, there exists a finite time $t_{1} \in[0, T)$ such that

$$
\begin{aligned}
& \lambda(t)=\lambda_{1}, \\
& A(t) \geq 2 \eta_{2},
\end{aligned}
$$

$$
\forall t \in\left[t_{1}, T\right)
$$

By (30), it can be concluded that, for any time $t \in\left[t_{1}, T\right)$, the following Ito differential inequality holds:

$$
L V_{0} \leq-\frac{\lambda_{1}}{2} A B V_{0}+\frac{1}{2}\left(\eta_{2}-\mu_{1}\left(r_{2}+r_{4}\right)\right)
$$

According to (34), the inequality with $V_{0}(\alpha, \beta)$ on time interval $t \in\left[t_{1}, T\right)$ can be obtained:

$$
\begin{aligned}
V_{0}(\alpha, \beta) \leq & \frac{\left(\eta_{2}-\mu_{1}\left(r_{2}+r_{4}\right)\right)}{\lambda_{1}} \\
& +V_{0}(\alpha, \beta) e^{\left(-\left(\lambda_{1} / 2\right)\left(t-t_{1}\right)\right)}
\end{aligned}
$$

By observing (35), it is easy to infer that $V_{0}(\alpha, \beta)$ is bounded on $t \in\left[t_{1},+\infty\right)$ and continuous on $t \in\left[0, t_{1}\right]$; then it can be generalized that $V_{0}(\alpha, \beta)$ is bounded on $t \in[0, T)$. Therefore, two cases of time $T$ need to be discussed.

The first case: $T<+\infty$.

If $V_{0}(\alpha, \beta)=h_{1}>0$ when $t \in\left[t_{1}, T\right]$, then it can obtain the following inequality according to (34):

$$
\begin{aligned}
\frac{\lambda_{1} h_{1}}{2} \int_{t_{1}}^{T} B(s) d s \leq & \frac{1}{2}\left(\eta_{2}-\mu_{1}\left(r_{2}+r_{4}\right)\right)\left(T-t_{1}\right) \\
& +V(\alpha, \beta)
\end{aligned}
$$

By observing (36) and (14), simultaneously, using $\alpha_{1}=e_{1} /$ $(A B)^{b}$ and $\beta_{1}=\widehat{z}_{1} /(A B)^{b}$, one has

$$
\dot{A}(t)=\frac{A}{(A B)^{2 b}}\left(\widehat{z}_{1}^{2}+e_{1}^{2}-\delta^{2}\right) \leq \frac{\lambda_{1} h_{1}}{2} B
$$

According to (36) and (37) again, one can get

$$
A(T)-A(0) \leq \frac{\lambda_{1} h_{1}}{2} \int_{t_{1}}^{T} B(s) d s
$$

At the moment, by observing (38), it can find that $A(T)-$ $A(0)=+\infty$ on the left and $\left(\lambda_{1} h_{1} / 2\right) \int_{t_{1}}^{T} B(s) d s$ is constant on the right, which contradicts (38). Therefore, it is a wrong judgment that conclusion (32) does not hold.

If $V_{0}(\alpha, \beta)=0$ when $t \in\left[t_{1}, T\right]$, by definition $\sigma$ as any positive constant and according to $\lim _{t \rightarrow T} A(t) B(t)=+\infty$, there exists $t_{2} \geq t_{1}$ such that, $\forall t \in\left[t_{2}, T\right)$, it can obtain

$$
A(t) B(t) \geq \frac{\eta_{2}}{\sigma \lambda_{1}}
$$

In addition, since $V_{0}(\alpha, \beta)=0$, there exists $t_{3} \geq t_{2}$ such that $V_{0}\left(\alpha\left(t_{3}\right), \beta\left(t_{3}\right)\right)<\sigma / 2$. Through the above analysis, (34) can be converted to

$$
L V_{0} \leq-\frac{2 \eta_{2}}{\sigma} V_{0}+\frac{1}{2}\left(\eta_{2}-\mu_{1}\left(r_{2}+r_{4}\right)\right), \quad \forall t \in\left[t_{3}, T\right)
$$


According to (40), the inequality with $V_{0}(\alpha, \beta)$ on time interval $t \in\left[t_{3}, T\right)$ can be obtained:

$$
\begin{aligned}
V(\alpha, \beta) \leq & \frac{\sigma}{2}+\frac{1}{2}\left(\eta_{2}-\mu_{1}\left(r_{2}+r_{4}\right)\right) \\
& +V\left(\alpha\left(t_{3}\right), \beta\left(t_{3}\right)\right) \\
\leq & \sigma+\frac{1}{2}\left(\eta_{2}-\mu_{1}\left(r_{2}+r_{4}\right)\right)
\end{aligned}
$$

and this proves that $\lim _{t \rightarrow T} V_{0}(\alpha, \beta)=0$.

Suppose the following inequality holds:

$$
\dot{A}(t) \leq \vartheta_{1}(t) B(t), \quad t \in[0, T)
$$

where $\vartheta_{1}(t)=\left(\alpha_{1}^{2}+\beta_{1}^{2}-\delta^{2} / 2(A B)^{2 b}\right)^{2}, t \in[0, T)$. By (4), (14), and (16), the following can be obtained:

$$
x_{1}=(A B)^{b}\left(\alpha_{1}+\beta_{1}\right)+y_{r}
$$

and, correspondingly, one has

$$
\left|x_{1}(t)\right| \leq \eta+\vartheta_{2}(t)(A B)^{b}
$$

where $\vartheta_{2}(t)=\left|\alpha_{1}(t)+\beta_{1}(t)\right|$. By (14) and Young' inequality, one can obtain

$$
\dot{B} \leq \mu_{2} B\left(1+\left|x_{1}\right|^{p}\right)^{2} \leq 2 \mu_{2} B\left(1+\eta+\vartheta_{2}(t)(A B)^{b}\right)^{2 p}
$$

and after further simplification one has

$$
\begin{array}{rl}
2 \mu_{2} & B\left(\eta+\vartheta_{2}(t)(A B)^{b}\right)^{2 p} \\
& \leq 2^{2 p} \mu_{2} \eta^{2 p-1} B+2^{2 p} \mu_{2} \vartheta_{2}^{2 p-1} A B^{2}
\end{array}
$$

Then (46) can be converted to

$$
\begin{array}{rl}
\dot{B}(t) \leq\left(2 \mu_{2}+2^{2 p} \mu_{2} \eta^{2 p-1}\right) B+2^{2 p} \mu_{2} \vartheta_{2}^{2 p-1} A B^{2} & \\
t & t \in[0, T)
\end{array}
$$

Next define the function $\Omega=A B V_{0}$ and then by (34), (42), (47), and Ito differential theorem, one has

$$
\begin{aligned}
L \Omega & \leq-A B\left(\frac{2 \eta_{2}}{\sigma} V_{0}+\frac{1}{2}\left(\eta_{2}-\mu_{1}\left(r_{2}+r_{4}\right)\right)\right) \\
& \leq-A B\left(\left(\frac{2 \eta_{2}}{\sigma}-\vartheta_{1}(t)-2^{2 p} \mu_{2} \vartheta_{2}^{2 p-1}\right) V_{0}\right. \\
& \left.+\frac{1}{2}\left(\eta_{2}-\mu_{1}\left(r_{2}+r_{4}\right)\right)\right)
\end{aligned}
$$

According to (42) and (47), it can be concluded that there exists $t_{4} \geq t_{1}$ such that the following inequality can hold:

$$
2^{2 p} \mu_{2} \vartheta_{2}^{2 p-1} \leq \frac{\eta_{2}}{\sigma}-\vartheta_{1}
$$

Further, it can get

$$
L \Omega \leq-A B\left(\frac{\eta_{2}}{\sigma} \Omega-\frac{1}{2}\left(\mu_{1}\left(r_{2}+r_{4}\right)-\eta_{2}\right)\right), \quad t \geq t_{4}
$$

which shows that $\Omega$ is bounded. According to the definition of $\vartheta_{1}(t)=\left(\alpha_{1}^{2}+\beta_{1}^{2}-\delta^{2} / 2(A B)^{2 b}\right)^{2}, t \in[0, T)$ and through inequality amplification, one has

$$
(A B)^{2 b}\left(e_{1}^{2}+\widehat{z}_{1}^{2}\right) \leq \frac{\lambda_{1} \lambda_{2} h_{1}}{2}
$$

which can prove that $\lim _{t \rightarrow T}\left(\widehat{z}_{1}^{2}+e_{1}^{2}\right)=0$ holds. Thence, there exists $t_{5}$ such that, for $t \in\left[t_{5}, T\right)$, it can obtain

$$
\widehat{z}_{1}^{2}(t)+e_{1}^{2}(t) \leq \frac{\lambda_{1} \lambda_{2} h_{1}}{2(A B)^{2 b}} \leq \frac{\delta^{2}}{2}
$$

By (52) and (14), it can get $\dot{A}(t)=0, t \in\left[t_{5}, T\right)$ which obviously conflicts with description of (14). Therefore, this is a false assertion that conclusion (32) does not hold.

The second case: $T=+\infty$.

Definition $\sigma$ is any positive constant; by $\lim _{t \rightarrow T} A(t) B(t)=$ $+\infty$ it can be seen that there exist $t_{2} \geq t_{1}$ such that, $\forall t \in$ $\left[t_{2}, T\right)$, one has

$$
\begin{aligned}
A(t) B(t) & \geq \frac{2 \eta_{2}}{\sigma \lambda_{1}} \Longrightarrow \\
-A(t) B(t) \lambda_{1} & \leq-\frac{2 \eta_{2}}{\sigma}
\end{aligned}
$$

Then (34) can be converted to

$$
L V_{0} \leq-\frac{2 \eta_{2}}{\sigma} V_{0}+\frac{1}{2}\left(\eta_{2}-\mu_{1}\left(r_{2}+r_{4}\right)\right), \quad \forall t \geq t_{2}
$$

By (53) and (54), the following can be obtained:

$$
\begin{aligned}
V_{0}(\alpha, \beta) \leq & \frac{1}{2}\left(\eta_{2}-\mu_{1}\left(r_{2}+r_{4}\right)\right) \\
& +V\left(\alpha\left(t_{2}\right), \beta\left(t_{2}\right)\right) e^{\left(-\left(2 \eta_{2} / \sigma\right)\left(t-t_{2}\right)\right)}, \\
& \quad t \geq t_{2}
\end{aligned}
$$

and then there exists $t_{3} \geq t_{1}$ such that the following inequality holds:

$$
\begin{aligned}
& V_{0}\left(\alpha\left(t_{2}\right), \beta\left(t_{2}\right)\right) e^{\left(-\left(2 \eta_{2} / \sigma\right)\left(t-t_{2}\right)\right)} \\
& \quad \leq \frac{1}{2}\left(\eta_{2}-\mu_{1}\left(r_{2}+r_{4}\right)\right), \quad \forall t \geq t_{3}
\end{aligned}
$$

Accordingly, it can obtain

$$
V(\alpha, \beta) \leq \eta_{2}-\mu_{1}\left(r_{2}+r_{4}\right), \quad \forall t \geq t_{3}
$$

Summarize the above analysis and it can be considered that $\lim _{t \rightarrow T} V_{0}(\alpha, \beta)=0$, then available $\dot{A}(t)=0$, which is also contradictory with (14).

Therefore, through the above two cases of analysis, it is a false assertion that conclusion (32) does not hold. Similarly, hypothetical judgment (32) is correct and system gain $A$ is bounded on $t \in[0, T)$ which satisfies

$$
\lim _{t \rightarrow T} A(t) \in\left[\frac{1}{\mu},+\infty\right)
$$

Next, we discuss boundedness for states $\alpha, \beta$ on $[0, T)$. 
By (24), (29), and Ito differential theorem, define function

$$
V_{2}(\beta)=\beta^{T} Q \beta
$$

and then one has

$$
\begin{aligned}
L V_{2} & \operatorname{Tr}\left(G^{T} P G\right)-2 A B|\beta|^{2}+2 A B \alpha_{1} \beta^{T} Q h \\
& -\left(\frac{\dot{A} B+A \dot{B}}{A B}\right) \beta^{T}\left(C_{b} Q+Q C_{b}\right) \beta \\
& +\|P\|\left(1+\left|x_{1}\right|^{p}\right)^{2}(|\alpha|+|\beta|+1)^{2}\left(c\left(\eta+\frac{d}{c}\right)\right)^{2} \\
\leq & -A B|\beta|^{2}+A B \alpha_{1}^{2}-\frac{\dot{B}}{B} \beta^{T}\left(C_{b} Q+Q C_{b}\right) \beta \\
& +\|P\|\left(1+\left|x_{1}\right|^{p}\right)^{2}(|\alpha|+|\beta|+1)^{2}\left(c\left(\eta+\frac{d}{c}\right)\right)^{2} \\
\leq & -A B|\beta|^{2}+A B \alpha_{1}^{2}+\mu_{1} c_{3} B|\beta|^{2} \\
& -\mu_{2} c_{4}\left(1+\left|x_{1}\right|^{p}\right)^{2}|\beta|^{2} \\
& +\|P\|\left(1+\left|x_{1}\right|^{p}\right)^{2}(|\alpha|+|\beta|+1)^{2}\left(c\left(\eta+\frac{d}{c}\right)\right)^{2}
\end{aligned}
$$

By (60) and choosing the appropriate parameter $\mu_{1}$, it gets

$$
L V_{2}
$$

$$
\begin{aligned}
\leq & -B|\beta|^{2}+A B \alpha_{1}^{2} \\
& +\|P\|\left(1+\left|x_{1}\right|^{p}\right)^{2}(|\alpha|+|\beta|+1)^{2}\left(c\left(\eta+\frac{d}{c}\right)\right)^{2} \\
\leq & -B\left(A-\left(2 \eta_{1}+\mu_{1} r_{2}+\mu_{1} r_{4}\right)\right)|\beta|^{2}+\eta_{1}+A B \alpha_{1}^{2}
\end{aligned}
$$

and then by (14), one has

$$
\begin{aligned}
\alpha_{1}^{2} & \leq\left(\alpha_{1}^{2}+\beta_{1}^{2}-\frac{\delta^{2}}{2(A B)^{2 b}}\right)+\frac{\delta^{2}}{2(A B)^{2 b}} \\
& \leq\left(\alpha_{1}^{2}+\beta_{1}^{2}-\frac{\delta^{2}}{2(A B)^{2 b}}\right)^{2}+\frac{\delta^{2}}{2}+1
\end{aligned}
$$

such that the following inequality can be derived:

$$
A B \alpha_{1}^{2} \leq A \dot{A}+A B\left(1+\frac{\delta^{2}}{2}\right)
$$

Thence, the following can be obtained:

$$
A B \alpha_{1}^{2} \leq A \dot{A}+B \mu_{3}
$$

where $\mu_{3}=A\left(1+\delta^{2} / 2\right)$. By (64) and (61), the following can be derived:

$$
L V_{2} \leq-B\left(A-\left(2 \eta_{1}+\mu_{1} r_{2}+\mu_{1} r_{4}\right)\right)|\beta|^{2}+A \dot{A}+\mu_{3} B
$$

According to the comparison theorem, one has

$$
\begin{gathered}
|\beta|^{2} \leq \mu_{3} B+\left(V_{2}\left(\beta_{0}\right)+\left(A^{2}(t)-A(0)^{2}\right)\right) \\
\cdot\left(2 \eta_{1}+\mu_{1} r_{2}+\mu_{1} r_{4}\right)
\end{gathered}
$$

It has been shown above that $A$ is bounded on $[0, T)$. Further, (66) can prove that $\beta$ is bounded on $[0, T)$.

According to the description of system (17), the state transition is introduced again:

$$
\Pi_{i}=\frac{1}{(\bar{A} B)^{i-1+b}} e_{i}
$$

where $\bar{A}=2 \bar{c}^{2}\|P\|^{2} / c_{1} \mu_{2}$.

Then the system error equation is converted to

$$
\begin{aligned}
\dot{\Pi}= & \bar{A} B L \Pi+\bar{A} B h \Pi_{1}-A B \Gamma h \Pi_{1}-\frac{\dot{B}}{B} C_{b} \Pi \\
& +\bar{G}(z, u, \bar{A}, B)
\end{aligned}
$$

where $\bar{G}=\left(g_{1} /(\bar{A} B)^{b}, g_{2} /(\bar{A} B)^{b+1}, \cdots, g_{n} /(\bar{A} B)^{b+n-1}\right)^{T}, \Gamma=$ $\operatorname{diag}\left(1, A / \bar{A}, \cdots,(A / \bar{A})^{n-1}\right)$.

Define Lyapunov function

$$
V_{3}(\Pi)=\Pi^{T} P \Pi
$$

and according to Ito's theorem, the trajectory of (69) along the system (68) is

$$
\begin{aligned}
L V_{3} \leq & -\bar{A} B|\Pi|^{2}+2 \Pi_{1} \bar{A} B h^{T} P \Pi+2 \bar{G} P \Pi \\
& -2 \Pi_{1} A B h^{T} \Gamma P \Pi-\frac{\dot{B}}{B} \Pi^{T}\left(C_{b} P+P C_{b}\right) \Pi \\
\leq & -\bar{A} B|\Pi|^{2}+2 \Pi_{1} \bar{A} B h^{T} P \Pi-2 \Pi_{1} A B h^{T} \Gamma P \Pi \\
& +2 \bar{G}^{T} P \Pi+\mu_{1} c_{2} B|\Pi|^{2} \\
& -\mu_{2} c_{1}\left(1+\left|x_{1}\right|^{p}\right)^{2}|\Pi|^{2}
\end{aligned}
$$

Furthermore, by the inequalities

$$
\begin{aligned}
& \left|2 \Pi_{1} \bar{A} B h^{T} P \Pi\right| \leq \bar{A}^{2} B\left\|h^{T} P\right\|^{2} \Pi_{1}^{2}+B|\Pi|^{2} \\
& 2 \Pi_{1} A B h^{T} \Gamma P \Pi \leq A^{2} B|h|^{2}\|\Gamma P\|^{2} \Pi_{1}^{2}+B|\Pi|^{2}
\end{aligned}
$$

(70) can be converted to

$$
L V_{3} \leq-B|\Pi|^{2}+\ell B \Pi_{1}^{2}+\ell B|\beta|^{2}+\ell
$$

where $\ell$ is unknown constant. Simultaneously, by (63) and (64), one has

$$
L V_{3} \leq-\lambda_{1} B V_{3}+\ell A \dot{A}+\ell B\|\beta\|^{2}+\ell\left(1+\mu_{3}\right)
$$

Since the above analysis has proved that $\beta$ is bounded on $[0, T)$, then $(73)$ can be converted to

$$
L V_{3} \leq-\lambda_{1} B V_{3}+\ell A \dot{A}+\bar{\ell} B
$$


where $\bar{\ell}=\ell \max \left(\sup \|\beta\|^{2}, 1+\mu_{3}\right)$. Simultaneously, by (16) and (17), one has

$$
\begin{array}{r}
\frac{1}{\lambda_{1}}\|\Pi\|^{2} \leq V_{3}\left(\Pi_{0}\right)+\frac{\bar{\ell}}{\lambda_{1}}+\ell\left(\frac{\left(A^{2}(t)-A(0)^{2}\right)}{2}\right) \\
t \in[0, T)
\end{array}
$$

Since the above analysis has proved that $A$ is bounded on $[0, T)$, then it can be inferred that $\Pi$ is bounded on $[0, T)$, Furthermore, combined with the above conclusions, it can be obtained that $\alpha$ is bounded on $[0, T)$.

Finally, we discuss the boundedness of state $B$ on $[0, T)$.

Since it has been concluded that system states $\alpha$ and $\beta$ are bounded on $[0, T)$, then it can obtain

$$
\left|\alpha_{1}+\beta_{1}\right| \leq k
$$

where $k$ is positive constant and depends on the state initial value $\alpha(0), \beta(0)$. By (14), it can deduce

$$
\dot{B} \leq-\mu_{1} B^{2}+2 \mu_{2} B+2 \mu_{2} \bar{c}^{2 p} B
$$

Using Young's inequality, (77) can be split to get the following relation form:

$$
\begin{array}{r}
2 \mu_{2} B \leq E B^{2}+C \\
2 \mu_{2} \bar{c}^{2 p} B \leq F B^{2}+D
\end{array}
$$

where $C, D, E, F$ are suitable coefficients or parameters.

It is equivalent to

$$
\dot{B} \leq \bar{E} B^{2}+\bar{C}
$$

where $\bar{C}, \bar{E}$ are suitable coefficients or parameters again. According to (67), it can be concluded that the state $B$ is bounded on $[0, T)$.

In summary, system high-gains $A, B$ and states $\alpha, \beta$ are all bounded on $[0, T)$. The boundedness of the system has been judged.

3.4. Output Feedback Tracking Controller Design. Based on the analysis of above, this section first gives the output feedback tracking controller.

Theorem 5. Consider a class of stochastic nonlinear system (4) whose output is $y=x_{1}-y_{r}$ under Assumptions 2 and 3 and then the following output feedback practical tracking controller can be designed:

$$
u=-\left((A B)^{n} k_{1} \widehat{z}_{1}+\cdots+(A B) k_{n} \widehat{z}_{n}\right)
$$

where $A, B$ are defined by (14); $k_{i}>0$ is coefficient of Hurwitz polynomial $s^{n}+h_{1} s^{n-1}+\cdots+h_{n-1} s+h_{n}$ with $h_{i}=k_{n-i+1}$. Then, for any $\delta>0$, there exists a finite time $T$ such that $\left|x_{1}-y_{r}\right| \leq \delta, \forall t \geq T$, and all the states of stochastic nonlinear system (1) and (13) are bounded on $[0, T)$ under the action of output feedback practical tracking controller (80).

The proof process of Theorem $\mathbf{5}$ is given.
Using the inequality theorem

$$
\left|\max (x, 0)^{2}-\max (y, 0)^{2}\right| \leq|x-y|^{2}
$$

It can be easily inferred that

$$
\frac{B}{(A B)^{4 b}}\left(z_{1}^{2}+e_{1}^{2}-\frac{\delta^{2}}{2}\right)^{2}, \quad x, y \in R
$$

is consistently continuous; that is, $\dot{A}$ is consistent and continuous.

Since system high-gains $A, B$ and states $\alpha, \beta$ are all bounded on $[0, T)$, it is easy to conclude that $\dot{e}, \dot{\vec{z}}, \dot{A}, \dot{B}$ are also bounded on $[0, T)$. According to the Barbalat lemma in [28], one has $\lim _{t \rightarrow+\infty} \dot{A}(t)=0$. By the above analysis, it can be deduced that there exists $T$ such that

$$
\left(e_{1}^{2}(t)+\widehat{z}_{1}^{2}(t)\right)^{1 / 2} \leq \text { CONSTANT }, \quad t \geq T
$$

Therefore, it can also be inferred that

$$
\left|x_{1}-y_{r}\right|=\left|z_{1}\right| \leq\left|e_{1}\right|+\left|\widehat{z}_{1}\right| \leq \delta, \quad t \geq T
$$

The proof of Theorem 5 is over such that output feedback practical tracking controller design is achieved.

\section{Simulation Example}

This section considers two-dimensional stochastic nonlinear systems as described below which are used extensively in offshore engineering valve models to verify the output feedback practical tracking controller designed in Section 3:

$$
\begin{aligned}
d x_{1} & =x_{2} d t \\
d x_{2} & =u d t-\sigma_{1}\left(1+x_{1}^{2}\right)\left|x_{2}\right|^{\sigma_{2}} d \omega \\
y & =x_{1}-y_{r}
\end{aligned}
$$

where $\sigma_{1}, \sigma_{2}$ are unknown parameters. System (85) satisfies Assumptions 2 and 3 with the corresponding parameters $\eta_{1}=$ $\sigma_{1} \sigma_{2}, \eta_{2}=\sigma_{1}\left(1-\sigma_{2}\right), p=2 . \omega$ is the $m$-dimensional standard Brownian motion defined on the probability space $(\Omega, \Gamma, \mathrm{P})$, which $\Omega$ is the sample space, $\Gamma$ is the algebra, and $\mathrm{P}$ is the probability measure. The given tracking trajectory is $y_{r}(t)=$ $1.5 \sin (2 t)\left(1-e^{-0.05 t^{3}}\right)$.

In numerical simulation, define $f_{1}(x)=0, f_{2}(x)=$ $-\sigma_{1}\left(1+x_{1}^{2}\right)\left|x_{2}\right|^{\sigma_{2}}$ and then, using inequality transform, it can get

$$
\left|f_{2}\right| \leq\left(1+x_{1}^{2}\right)\left(\sigma_{1} \sigma_{2}\left|x_{2}\right|+\sigma_{1}\left(1-\sigma_{2}\right)\right)
$$

Next start the system simulation and select the initial value and related parameters:

$$
\begin{aligned}
x_{1}(0) & =0.035, \\
x_{2}(0) & =-2.5, \\
\widehat{z}_{1}(0) & =0, \\
\widehat{z}_{2}(0) & =0, \\
\delta & =0.5, \\
h_{1} & =2,
\end{aligned}
$$




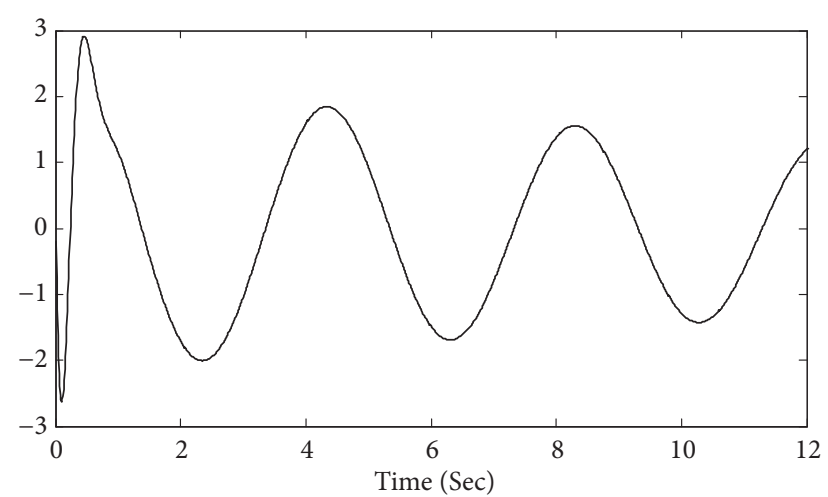

FIGURE 1: The trajectory of system state $x_{1}$.

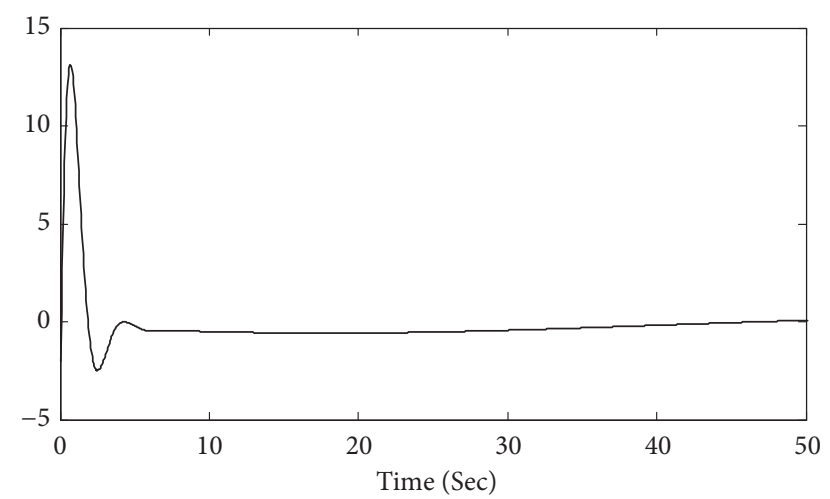

FIGURE 2: The trajectory of system state $x_{2}$.

$$
\begin{aligned}
& h_{2}=5, \\
& k_{1}=45, \\
& k_{2}=55, \\
& \mu_{1}=10, \\
& \mu_{2}=20,
\end{aligned}
$$

Figures 1-3 show the response characteristics of the closedloop system (85). By observing the simulation graph, the practical states of the two-dimensional system (85) are bounded and gradually converged. Simultaneously, it can be observed that the output tracking error of the system gradually converges after $2 \mathrm{~s}$ and finally adjusts to near zero. The results verify the effectiveness of the designed output feedback practical tracking controller.

\section{Conclusion and Future Prospects}

In this paper, we study the tracking problem of output feedback for a class of stochastic nonlinear systems which remove the more stringent growth conditions and satisfy polynomial function growth conditions. The parameters of the output polynomial function growth conditions are

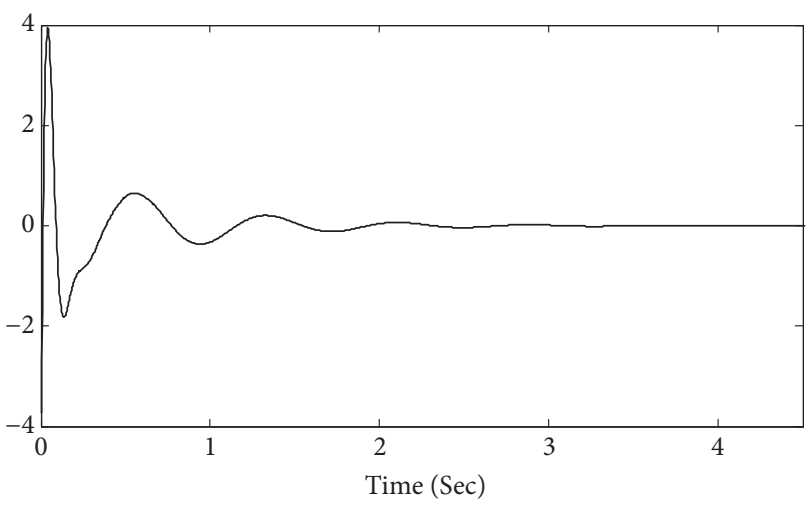

FIGURE 3: The trajectory of system output $y$.

unknown which leads to the uncertainty of the parameters and generalizes the previous output feedback practical tracking control research results. Finally, an output feedback practical tracking controller with dynamically updated gains is constructed explicitly so that all the states of the closed-loop systems are globally bounded and the tracking error belongs to arbitrarily small interval after some positive finite time. A numerical example illustrates the efficiency of the theoretical results.

This paper has made some valuable research results, but also produced some further in-depth research of problem. We consider whether it can generalize it to stochastic nonlinear systems with uncertain control coefficients and expand it to time-delay systems. How to design the output feedback practical tracking controller for this type of extended system? It is worth studying the problem.

\section{Data Availability}

No data were used to support this study.

\section{Conflicts of Interest}

The author declares that there are no conflicts of interest regarding the publication of this paper.

\section{Acknowledgments}

This work is financially supported by Natural Science Foundation of Zhejiang Province of China (no. LQ18F030007).

\section{References}

[1] B. Yang and W. Lin, "Robust output feedback stabilization of uncertain nonlinear systems with uncontrollable and unobservable linearization," Institute of Electrical and Electronics Engineers Transactions on Automatic Control, vol. 50, no. 5, pp. 619-630, 2005.

[2] H. Lei and W. Lin, "Universal adaptive control of nonlinear systems with unknown growth rate by output feedback," Automatica, vol. 42, no. 10, pp. 1783-1789, 2006. 
[3] L. Praly and Z. P. Jiang, "Linear output feedback with dynamic high gain for nonlinear systems," Systems \& Control Letters, vol. 53, no. 2, pp. 107-116, 2004.

[4] A. Astolfi and L. Praly, "Global complete observability and output-to-state stability imply the existence of a globally convergent observer," Mathematics of Control, Signals, and Systems, vol. 18, no. 1, pp. 32-65, 2006.

[5] L. Praly, "Asymptotic stabilization via output feedback for lower triangular systems with output dependent incremental rate," Institute of Electrical and Electronics Engineers Transactions on Automatic Control, vol. 48, no. 6, pp. 1103-1108, 2003.

[6] G. Kaliora, A. Astolfi, and L. Praly, "Norm estimators and global output feedback stabilization of nonlinear systems with ISS inverse dynamics," Institute of Electrical and Electronics Engineers Transactions on Automatic Control, vol. 51, no. 3, pp. 493-498, 2006.

[7] P. Krishnamurthy and F. Khorrami, "Global adaptive output feedback tracking for nonlinear systems linear in unmeasured states," in Proceedings of the 2001 American Control Conference, pp. 4814-4819, June 2001.

[8] M. Polyearpou and A. loannou P, "A robust adaptive nonlinear control design," in Proceedings of the 1993 American Control Conference, pp. 1365-1369, San Francisco, Calif, USA, 1993.

[9] P. Krishnamurthy, F. Khorrami, and R. S. Chandra, "Global high-gain-based observer and backstepping controller for generalized output-feedback canonical form," Institute of Electrical and Electronics Engineers Transactions on Automatic Control, vol. 48, no. 12, pp. 2277-2284, 2003.

[10] P. Krishnamurthy, F. Khorrami, and P. Jiang Z, "Global output feedback tracking for nonlinear systems in generalized outputfeedback canonical form," in Proceeding of the 2001 American Control Conference, pp. 4241-4246, IEEE Press, Arlington, 2001.

[11] L.-C. Guo, X. Zuo, J.-W. Liu, and H.-Q. Liang, "Outputfeedback control of a class of stochastic nonlinear systems with power growth conditions," International Journal of Control, Automation, and Systems, vol. 12, no. 2, pp. 274-282, 2014.

[12] F. Mazenc, L. Praly, and W. P. Dayawansa, "Global stabilization by output feedback: examples and counterexamples," Systems \& Control Letters, vol. 23, no. 2, pp. 119-125, 1994.

[13] W. Chen, L. Jiao, J. Li, and R. Li, "Adaptive NN backstepping output-feedback control for stochastic nonlinear strictfeedback systems with time-varying delays," IEEE Transactions on Systems, Man, and Cybernetics, Part B: Cybernetics, vol. 40, no. 3, pp. 939-950, 2010.

[14] K. Ezal, Z. Pan, and P. V. Kokotovic, "Locally optimal and robust backstepping design," Institute of Electrical and Electronics Engineers Transactions on Automatic Control, vol. 45, no. 2, pp. 260-271, 2000.

[15] L. Guo, J. Liu, X. Zuo, and H. Liang, "Output-Feedback Stabilization for a Special Class of Stochastic Nonlinear TimeDelay System With More General Growth Conditions," Journal of Dynamic Systems, Measurement, and Control, vol. 136, no. 6, article 061004, 2014.

[16] F. Yang and C. Wang, "Adaptive stabilization for nonholonomic mobile robots with uncertain dynamics and unknown visual parameters," Transactions of the Institute of Measurement and Control, vol. 37, no. 2, pp. 282-288, 2015.

[17] F. Shang, Y. Liu, and C. Zhang, "Adaptive output feedback stabilization for a class of nonlinear systems with inherent nonlinearities and uncertainties," International Journal of Robust and Nonlinear Control, vol. 21, no. 2, pp. 157-176, 2011.
[18] F. Shang, Y. Liu, and C. Zhang, "Adaptive output feedback control for a class of planar nonlinear systems," Asian Journal of Control, vol. 11, no. 5, pp. 578-586, 2009.

[19] X. Yan and Y. Liu, "Global practical tracking for high-order uncertain nonlinear systems with unknown control directions," SIAM Journal on Control and Optimization, vol. 48, no. 7, pp. 4453-4473, 2010.

[20] X. Yan and Y. Liu, "Global practical tracking by output-feedback for nonlinear systems with unknown growth rate," Science China Information Sciences, vol. 54, no. 10, pp. 2079-2090, 2011.

[21] X. Yan and Y. Liu, "The further result on global practical tracking for high-order uncertain nonlinear systems," Journal of Systems Science \& Complexity, vol. 25, no. 2, pp. 227-237, 2012.

[22] Q. Gong and C. Qian, "Global practical tracking of a class of nonlinear systems by output feedback," Automatica, vol. 43, no. 1, pp. 184-189, 2007.

[23] S.-J. Liu and J.-F. Zhang, "Output-feedback control of a class of stochastic nonlinear systems with linearly bounded unmeasurable states," International Journal of Robust and Nonlinear Control, vol. 18, no. 6, pp. 665-687, 2008.

[24] N. Duan and X.-J. Xie, "Further results on output-feedback stabilization for a class of stochastic nonlinear systems," Institute of Electrical and Electronics Engineers Transactions on Automatic Control, vol. 56, no. 5, pp. 1208-1213, 2011.

[25] X.-J. Xie and W.-Q. Li, "Output-feedback control of a class of high-order stochastic nonlinear systems," International Journal of Control, vol. 82, no. 9, pp. 1692-1705, 2009.

[26] W. Li, Y. Jing, and S. Zhang, "Output-feedback stabilization for stochastic nonlinear systems whose linearizations are not stabilizable," Automatica, vol. 46, no. 4, pp. 752-760, 2010.

[27] W. Li, X. Liu, and S. Zhang, "Output-feedback stabilization of high-order stochastic nonlinear systems with more general growth conditions," in Proceedings of the 30th Chinese Control Conference, CCC 2011, pp. 5953-5957, July 2011.

[28] H. K. Khalil, Nonlinear Systems, Publishing House of Electronics Industry, Beijing, China, 3rd edition, 2007. 


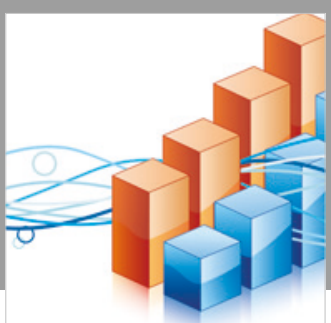

Advances in

Operations Research

\section{-n-m}
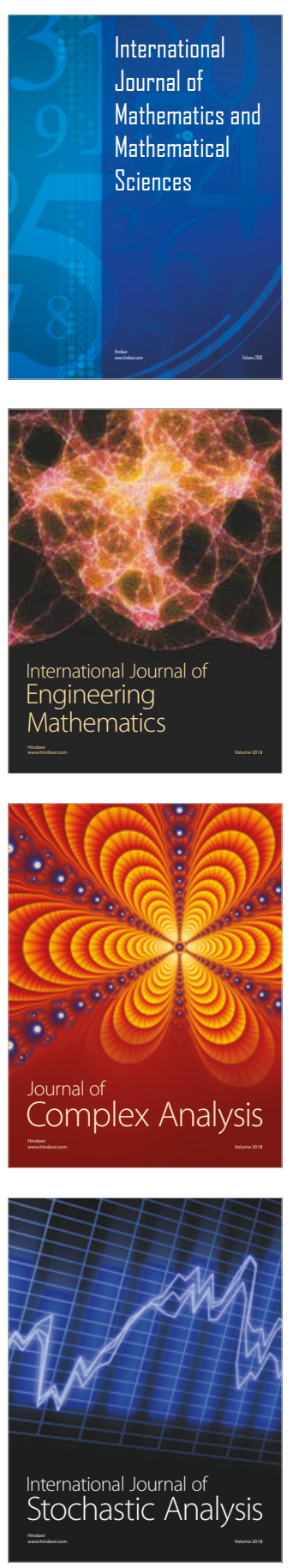
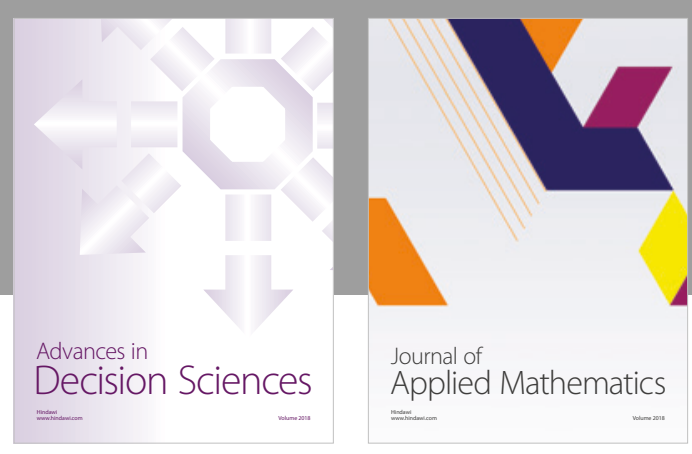

Journal of

Applied Mathematics
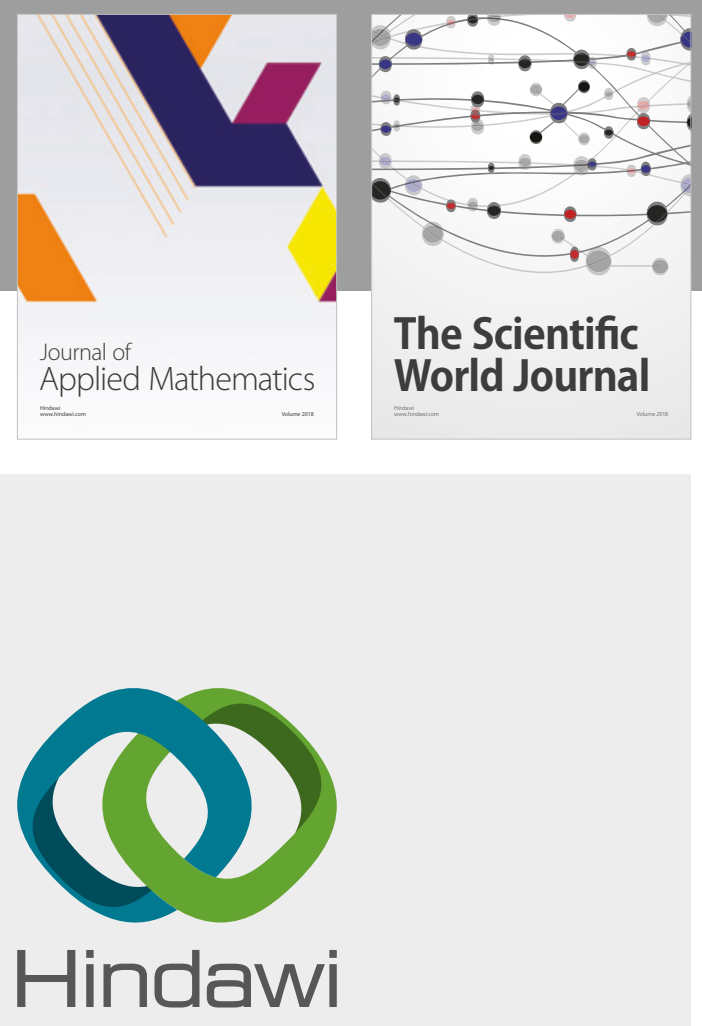

Submit your manuscripts at

www.hindawi.com

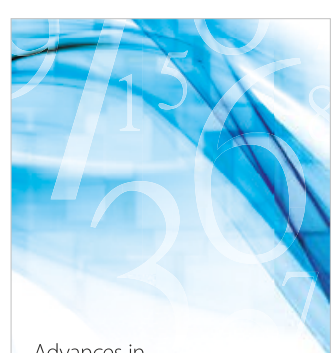

Advances in
Numerical Analysis
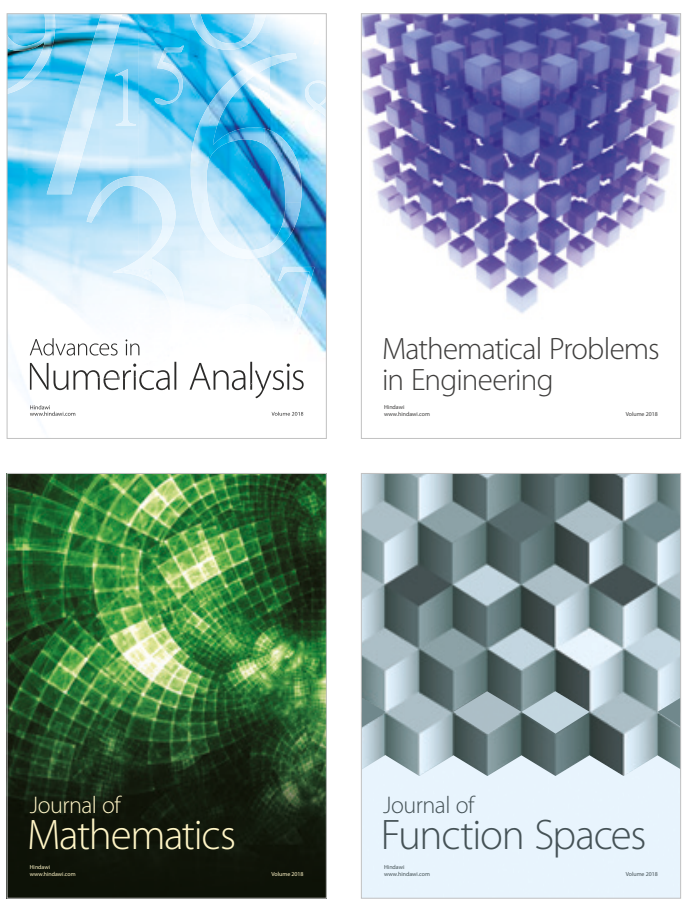

Mathematical Problems in Engineering

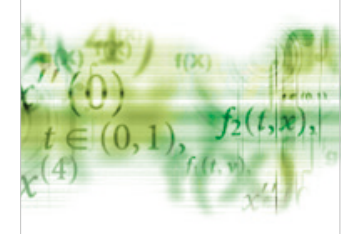

International Journal of

Differential Equations

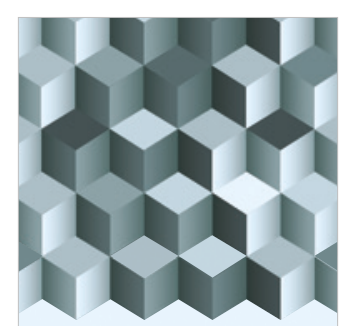

Journal of

Function Spaces

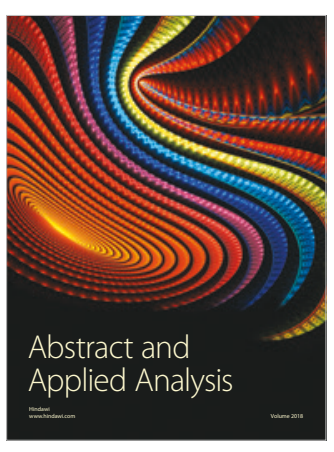

The Scientific

World Journal

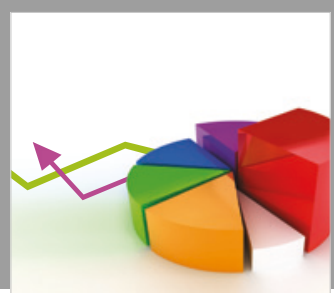

Journal of

Probability and Statistics
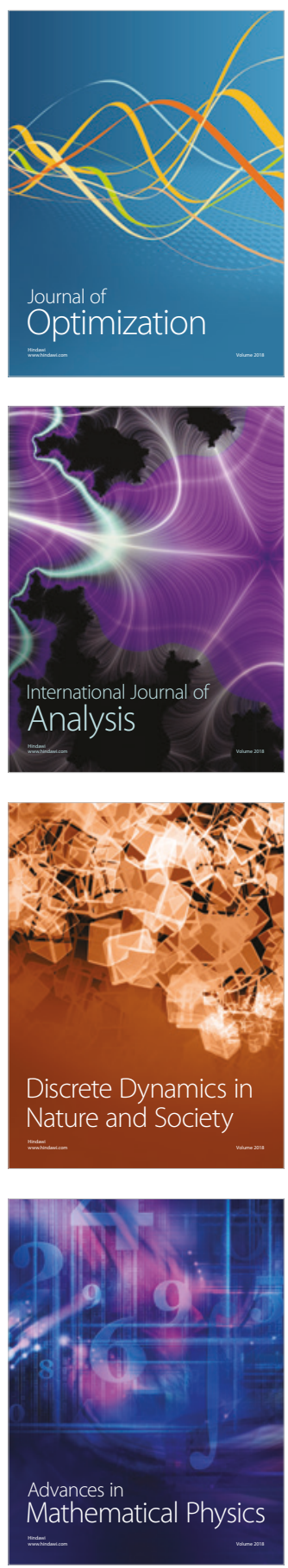